\title{
Turning Shortcomings into Challenges: Brain-Computer Interfaces for Games
}

\author{
Anton Nijholt, Boris Reuderink, and Danny Oude Bos \\ University of Twente, Faculty EEMCS, \\ P.O. Box 217, 7500 AE, Enschede \\ The Netherlands \\ anijholt@cs.utwente.nl
}

\begin{abstract}
In recent years we have seen a rising interest in brain-computer interfacing for human-computer interaction and potential game applications. Until now, however, we have almost only seen attempts where BCI is used to measure the affective state of the user or in neurofeedback games. There have hardly been any attempts to design BCI games where BCI is considered to be one of the possible input modalities that can be used to control the game. One reason may be that research still follows the paradigms of the traditional, medically oriented, BCI approaches. In this paper we discuss current BCI research from the viewpoint of games and game design. It is hoped that this survey will make clear that we need to design different games than we used to, but that such games can nevertheless be interesting and exciting.
\end{abstract}

Keywords: Brain-computer Interfacing, Multimodal Interaction, Game Design.

\section{Introduction}

Brain-Computer Interfacing $(\mathrm{BCI})$ is finding its way in human-computer interaction $[26,27]$. In this paper we discuss the use of BCI in game and game-like applications. These applications are not that different from medical or military BCI applications. Medical applications, aiming at providing handicapped patients with communication and movement skills, have seen many research efforts. But we can also say that gamers, soldiers or, in fact, anybody is handicapped, in the sense that they will meet situations where it is desirable to have more skills and communication means than are available when using the usual verbal and nonverbal interaction modalities. The circumstances in which they have to perform challenge their abilities to control the environment and can demand control that cannot be delivered by conventional modalities (e.g. speech, gaze, keyboard, mouse). 'Induced disability' or 'situational disability' are words used to describe these circumstances. In fact, everybody, handicapped or not, will meet situations, in particular situations where they have to compete with others, where they would benefit from extra communication or movement modalities. This is particularly true in games, sports and entertainment.

There are other reasons that make games, gamers and the game industry interesting for BCI research and development. In particular, gamers are early adaptors. They are 
quite happy to play with technology, to accept that great efforts have to be made in order to gain a sometimes minimal advantage, and they are used to the fact that games have to be mastered by training, allowing them to go from one level to the next level and to get a higher ranking than their competitors. We may also expect interest from software companies for BCI games. There are enormous numbers of gamers. Being the first to introduce a new type of game, a new game element or a new interaction modality may bring them enormous profits. This is certainly an impetus for industry to invest in research and development into brain-computer interfacing.

We can use information made available to us from brain activity to adapt the interface to the user or to issue commands to the interface. Brain activity, whether it is consciously controlled and directed by the user or 'just' recorded in order to obtain information about the user's affective state, should be modeled and embedded in more general models of interaction in order to provide appropriate adaptation, feedback and a context where brain activity information is one of the many multi-modal interaction modalities that are provided to the gamer. From the traditional BCI point of view this is quite an unusual approach. Generally, BCI researchers prefer or assume that the only activity a subject performs is brain activity while any other activity (as extreme but nevertheless realistic examples, blinking of an eye or facial muscle movement) disrupts the activity in which the researcher is interested. We are not directly interested in designing games for ALS patients or designing games for gamers who have to behave like ALS patients and are punished for moving. Hence, measuring and interpreting brain signals and providing feedback in a particular game context is an aim that needs to be pursued. Employing measured brain activity to be used in everyday-life-like (domestic) applications is the next step.

BCI with the aim of obtaining knowledge about a user's experience of the game and maybe to adapt the game (interface) to the gamer is an important research issue. There are already several European research projects devoted to defining and measuring the game experience with the aim to use this knowledge for designing future games and to adapt games to their users [9]. One particular form of game experience is 'immersion' or 'flow' [5]. It can be considered as the ultimate goal of a game designer, being able to cause a flow experience where a gamer enters a situation where increasing challenges are to be met by increasing skills (see Figure 1). The gamer becomes immersed in the game, forgetting about time and other reality. Until now research aiming to understand this flow experience has concentrated on using more traditional physiological information, attempting to derive a user's affective state from, for

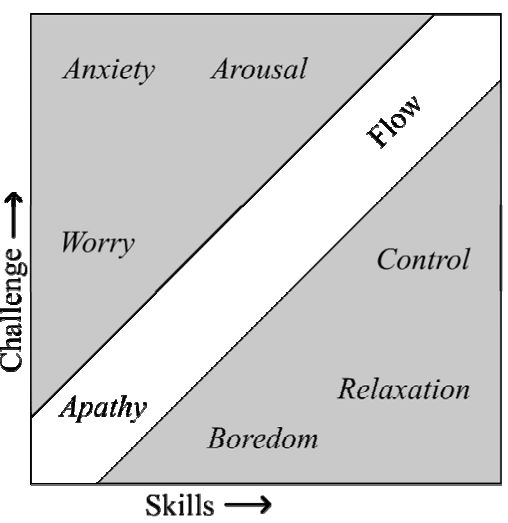

Fig. 1. Flow diagram, based on [5] example, heart rate, sweating, respiration, and blood pressure [10]. Recently, there have also been attempts to investigate how being in the flow can be read from body language [4]. It is certainly helpful if BCI 
can tell us a gamer is bored, anxious, or frustrated. Then it is clear that a gamer is not in the flow envisaged by the game designer.

Hence, measuring experience and affect and adapting a game to the affective state of the gamer is an important issue. But probably even more interesting are games and game environments that have been designed to allow or require control from brain activity that is consciously produced by a gamer or that has been evoked from external stimuli that have been integrated into a game. There can be game situations where such control is an added modality to the other means a gamer has to play the game. It may help or it may be the only possibility to reach a next level in a game.

This paper provides a state-of-the-art survey of the field of brain-computer interfacing as far as it is of interest from the point of view of games. With this survey in mind we discuss possibilities for including BCI as an added modality in game design and we discuss developments and challenges.

\section{BCI for Controlling and Adapting Games}

When we look at possible BCI games we are asking for BCI theory that allows us to distinguish and employ activity in different regions of the brain (using machine learning algorithms) and that allows us to map each of these activities to commands to control or adapt a game. When we look at game applications we need to take into account that gamers will prefer not to game in a MRI scanner, that gamers will not want to wear heavy head sets that measure their brain waves, and that not all gamers are yet willing to undergo surgery to have implants that will improve measuring their brain waves or improve their brain functions. There are some exceptions, but in this paper we assume that brain activity is measured using an EEG cap. Such a cap has electrodes attached to it that measure activity in different regions of the brain. We can 'read' such information and make it available to a game engine that controls the environment, to use to adapt the game to a recognized mental state of the user or to translate consciously produced activity to commands that allow a gamer to change the environment, to navigate, and to make decisions that allow him or her to survive in the game. We can distinguish different kinds of brain activity:

- the gamer is experiencing the game, the task and the interface, and gets, among other things, frustrated, engaged, irritated, bored or stressed; in particular it would be useful to measure whether a gamer is 'in the zone' or 'in the flow'; currently, flow experience research is empirical, asking users about their experiences; however, there are already attempts to read the 'flow experience' from the nonverbally displayed expressions by gamers, and BCI can help to adapt the interface in order to get a better matching of skills and challenges in the game;

- there are external stimuli (visual, auditory, tactile, ...) designed and generated by the game environment to force the user to choose among certain possibilities (i.e., make decisions in the game) or that occur in a more natural way because BCI recognizes that a gamer is interested in a particular event that happens during a game; such externally evoked potentials can be generated by the game environment (an unexpected obstacle is introduced) or by a gamer's opponent (asking a gamer to react on a particular sword movement); 
- the gamer consciously tries to evoke brain signals by performing a certain mental task; for instance, imagining a movement or doing a mental calculation, leading to brain signals that can be transformed in such a way that the application is controlled by this mental task, rather than by mouse, joystick or keyboard activity;

- the gamer consciously tries to control his or her global brain activity; that is, activity related to stress, attention, and relaxation, in order to control part of a game; this activity of the user or gamer is very much supported by performance feedback (visual or auditory) from the interface; this neurofeedback provides motivating rewards for the user and it stimulates the user to continue his or her efforts to control the game environment by thought.

\subsection{Internally Evoked Potentials}

We distinguish between internally and externally evoked brain signals. Motor imagery [20] is one of the possible ways in which we can have internally evoked potentials. In our motor cortex we can find a mapping from possible movements made by our body parts to brain regions in this cortex. That is, for example, imagining a movement of your left foot, imagining a movement of your index finger, or imagining a movement of the tip of your tongue, all these imaginary movements lead to distinguishable brain activity in the motor cortex of the brain. Moreover, imagining these movements leads to similar activity in similar or related regions of the motor cortex as executing or intending to execute such movements. By picking up these signals and translating them to commands, we can, for example, use brain activity to navigate in a virtual environment (see Figure 2), to guide our avatar, and also to add actions to our avatar or to the tools and weaponry used by our avatar in a game.

The mapping of 'thoughts' to actions in a virtual game environment does not necessarily have to be to 'natural'. A gamer can be asked to perform a difficult calculation (mental arithmetic) or to imagine a rotation of a geometric object. But preferably a required mental effort should be naturally embedded in a game because this helps a great deal to make the required game actions believable to the gamer and helps to keep the gamer immersed in the game. Mental efforts related to calculation or rotation, or other mental activities that have not yet been investigated, can become embedded in game environments. We can also look at more global levels of internally evoked brain activity. For example, changes in relaxation can be mapped on color changes in the environment and other changes in the landscape, or they influence the speed of changes in the environment, on the ability to move around, or on the fighting ability of game actors controlled by the gamer. Also, levels of relaxation can be mapped onto different game commands. However, generally we may expect that affect related brain signals are more useful to adapt the game to the particular gamer than to transform them to explicit commands that control game actions and events.

\subsection{Externally Evoked Potentials}

There are also many forms of externally evoked potentials that can be exploited by game designers. When looking at evoked potentials we should take into account what 
can be measured in a game situation. As an example, can we measure motor cortex brain activity that is evoked by looking at movements? On the other hand, it is well known that we can have externally evoked potentials in our brain. The stimuli that cause these potentials can be auditory (to be detected in the auditory cortex), visual (in the visual cortex), or somatosensory or tactile (in the somatosensory cortex), and combinations of these stimuli. Steady-state visually evoked potentials (SSVEPs), like flickering lights on a computer screen, have been used to allow a gamer to make binary decisions [16]. But there is no need to restrict ourselves to binary decisions since different frequencies cause different distinguishable brain activities. An example in with four SSVEP decision options are provided is shown in Figure 2.

Among the electrophysiological responses to external stimuli are also the eventrelated potentials (ERPs). While the evoked potentials reflect physical stimuli, the event-related potentials are more related to thoughts that are an automatic response to observed events. Hence, they are related to expectations and attention, and to changes in the mental state of the observer. A well-known example of these event-related potentials is the so-called P300 signal. Suppose you are playing a video game and you are anticipating certain events. Then, every time such an event takes place there is EEG measurable brain activity (a positive voltage deflection in the parietal cortex of the brain) about $300 \mathrm{~ms}$ after the stimulus onset. See Figure 2 for an applied example.
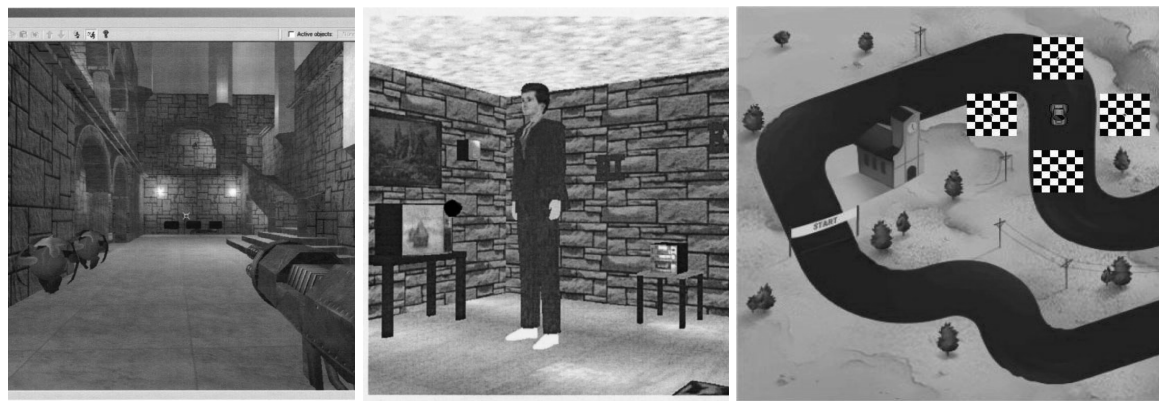

Fig. 2. Examples of games and virtual environments using imaginary movement [30], P300 [2], and SSVEP [21] paradigms respectively. To elicit the P300 potential, blinking spheres were connected with each controllable object in the room. For the SSVEP, each checkerboard was inverted at different frequencies. Imaginary movement is internally evoked, so no external stimuli are required for the interaction.

Less well-studied, but certainly interesting in the game context is the event-related N400 (a negative voltage deflection about $400 \mathrm{~ms}$ after the stimulus onset) effect. It has been studied in the context of experiencing unexpected events, for example, when we recognize that a word we assume to have recognized does not have a semantic fit in the particular sentence context [8]. Detecting such semantic incongruity has also been studied in the context of humor studies [1] since incongruity and incongruity resolution are important concepts in humor theory [6]. Obviously, in game applications it is important to be able to measure brain signals, such as the EEG measurable N400-like signals, that are related to surprise. 


\section{Games That Employ BCI}

In the previous section we mentioned various ways in which BCI can be employed in games. In research and demonstration contexts there are many attempts in which researchers 'play' with potential BCI game applications. Sometimes this is done for further developing ideas for research or medical applications. Game-like situations have also been designed to illustrate BCI research. Other reasons why researchers who aim at medical applications have looked at games are their potential for training environments for patients and to provide patients with entertainment facilities. Nonmedical applications are also becoming important and we now see that game companies are starting to do their own research.

\section{1 'Medical' Games}

In neurofeedback applications, EEG data is made available to the user in a graphical or auditory way with the aim to train the user to perform in a desired way. Desirable behavior leads to immediate rewards, undesirable behavior is discouraged. Neurofeedback is usually based on asking the user to control slow (or ultra-slow) brainwave activity. Generally, slow brain activity is associated with relaxation, drowsiness, or sleep. Training brainwave activity may make it easier to enter a state of alertness, or, on the other hand, make it easier to enter a relaxed state. Treating mental disorders, for example, Attention-Deficit/Hyperactivity Disorder (ADHD) in children, has been a driving force for this kind of research. And, obviously, having an engaging training environment will help to keep children spending time on training.

In [31], neurofeedback experiments with Nintendo and PlayStation games are reported. In their 'neurofeedback-modulated' games the game pad or joy stick becomes easier to control when the children produce the right brainwaves. As another example, in [36] a training environment is introduced, again for ADHD treatment, where children have to steer a ball to a particular target using control of 'slow cortical potential' brain activity. BCI together with commercial video game software has also been used to allow epileptic teenagers to play games such as Space Invaders.

There are many small companies that now introduce neurofeedback games for nonmedical applications on the market. We will return to these games in a later section.

\section{2 'Research' Games}

Simple and familiar video games have been given BCI control by researchers. The Berlin Brain-Computer Interface [14] has used motor imagery to play Pacman and Pong and similarly familiar games such as Tetris. Motor imagery applications exist for a First-Person Shooter game [30], navigating a ball in an environment where the ball has to jump over hills [24], navigating in Second Life [13] or other virtual environments, or controlling Google Earth [34]. Controlling the flippers of a virtual pinball machine by motor imagery also seems to be a promising application. A BCI game with brain activity related to real (finger) movements has been investigated in [18]. Externally evoked potentials have also been used in game-like implementations. Already in 1977 we saw the use of visually evoked potentials allowing the user to navigate in a maze [37]. Other examples are the control of a flight simulator [22] or 
the MindBalance game [16] where the user has to keep a tight-rope walker in balance. Some games that exploit more global brain activity have also been introduced. Wellknown examples in this category are games where the gamer is asked to control the movements of a ball, either on a table or in a virtual environment. Brainball is one of them. In this game, gamers have to control a ball on the table through their state of relaxation [12]. A similar game [19] uses both relaxation and stress related activity. In the same category we find games where the gamer is asked to control a tight-rope walker, similar to the tight-rope walker of [16], but now by controlling left and right hemisphere brain activity [35]. More examples can be found in the literature.

A probably useful observation, and we will return to it in section 4 , is that in many of these primitive games, we have visually rich $2 \mathrm{D}, 3 \mathrm{D}$, or virtual reality environments, and moreover, there were experiments that included the use of head-mounted devices (HMDs). Rather than finding a decrease in performance of the gamer, it was found that such environments were stimulating and engaging, resulting in a better performance. This can be concluded from experiments that involved the use of ERPs, in particular the use of P300 (see [2] and [3]), the use of VEPs, and the use of motor imagery [17] Experiments in such visually rich environments also made clear that VEPs can be used for multiple directional controls [21].

\subsection{Commercial Game Environments: Controlling the Game}

All of the examples in the previous section concern game ideas that have, for various reasons, been found useful in a research context. As has become clear from the short descriptions, in all these examples we have one type of brain signals that are used for one type of control and mostly there is one object that has to be controlled. A similar observation can nowadays be made for the early companies that have attempted to introduce BCI in game products, either for entertainment or for medical and health purposes. For example, there is a commercial variant of the Brainball game, meant to be used in technology exhibitions and museums, and there are now 'commercial' games that aim at monitoring and influencing the brain state of ADHD children.

More recently, however, we see large software companies such as IBM and Microsoft, large console game companies such as Sony and Nintendo, and smaller specialized companies such as Emotiv, NeuroSky, and OCZ entering and defining a (future) market for commercial BCI games and other non-medical applications. In particular (still) small companies such as Emotiv and NeuroSky aim at developing games that are more interesting and therefore more engaging than the 'one-trick' games mentioned above. Apart from having to aim at commercial success, and apart from having to obey technological constraints, a BCI game designer does not have to take into consideration the limitations of an ALS patient, limitations of someone who has to control a prosthetic device, or limitations associated with military applications.

What kinds of game applications can be expected from commercial game companies? Obviously, there are the neurofeedback games that aim at training a patient or a healthy user to perform better on certain mental activities. We will return to them in the next section. Maybe more interesting are companies that aim at controlling a game by brainwaves. 'Passive' brain activity that is present because of the (multimodal) perception of events in the environment can be used to adapt the game to the mental state associated with this activity. 'Active' brain activity, for 
example motor imagery, allows the user to control a game: for example, making decisions about actions his or her avatar has to perform in the game environment.

A demonstration game developed by Emotiv [7] asks the gamer to rebuild Stonehenge. By motor imagery, using a wireless headset, the gamer can push, rotate and lift giant stones in a $3 \mathrm{D}$ environment until the desired stone structure has been obtained. A next version is under development by the well-known game company Demiurge. In this game, mood, facial expressions, mental tasks and head movements are input modalities. Fourteen electrodes are positioned on the head with a futuristic looking headset. Head movements are measured with a built-in dual-axis gyroscope. In the game the gamer is a student of mental martial arts that can walk around in a virtual world and who learns how to manipulate objects in the world with thought commands and facial expressions (scare away an evil spirit). According to the affective state of the gamer the environment can be adapted, for example, colors can be changed, or the difficulty of the game tasks can be tailored to this state. Mapping between mental tasks and the thought commands is done during a short training phase. One issue that may pop up with these kinds of games is how to remember the mental tasks and perform them in a consistent way. In the case of movements it may help to actually perform the corresponding movements.

Other companies that work on these next-generation video games are Hitachi, EmSense and NeuroSky. The latter company's headset has only one electrode. NeuroSky is also looking at BCI controlled games for mobile phones. NeuroSky partners with Sega Toys. Games that are developed use the mouse for directional movement and BCI (levels of focus and relaxation) to manipulate objects. In a multiplayer version, a player can throw objects to other players.

\subsection{Commercial Game Environments: Neurofeedback Games}

People may have fun playing simple games such as Pacman, Space Invaders, Tetris, a virtual pinball machine, or similar brain-controlled games. From a commercial point of view, there is a growing market for these so-called 'casual' games, where a user interacts with his or her PDA, mobile phone, notebook or PC to enter and get involved in games that employ neurofeedback. These games are meant to train healthy users to control their brainwaves in order to get better performance. For example, they can be used in attention management training, which can be useful for professionals such as pilots or crisis managers, for activities such as driving, and for sportsmen preparing for their performance. While for some situations it is useful to enter a state of so-called 'absorbed attention' (for example an athlete preparing for a high jump), in other situations too much focus or 'attentional tunneling' may lead to hazardous situations. Consider for example a pilot who, in a stress situation, loses awareness of the global situation. Especially when looking at sports where concentration and relaxation are extremely important, like darts, golf, and archery, we can expect attention from companies that are developing neurofeedback games. And indeed, for some of these sports such training games have already been developed.

There are many games that ask a gamer for qualities similar to those that are needed for real-life professions and activities. In particular this is true when such activities have been made part of virtual reality simulation environments. People find it fun to perform in a car or flight simulator, compete with others during a virtual golf 
game, and improve their performance by whatever the simulation environment offers them, including auditory or visual aids that aim at training the control of their attention and other aspects of the quality of their performance. But also in the more violent individual and multi-player video games these issues are important and there are many gamers willing to improve their performance by implicit and natural neurofeedback during their game playing. While playing they unconsciously train the desired brain activity that will allow them to perform better in real life in the future.

Looking at the future of neurofeedback-modulated games, Pope and Palsson [31] argue that "Entertaining games that incorporate biofeedback in the background may offer a palatable and effective way to systematically guide the cerebral rewiring occurring during prolonged video game playing towards fostering creativity, concentration skills, precision motor skills, and other valuable abilities."

\section{Turn Shortcomings into Challenges}

In medical BCI applications we are looking at improving imperfect communication and bodily skills of patients by using technological tools in which BCI knowledge is incorporated. Certainly, there is no need to change this viewpoint when we want to look at non-medical and, in particular, game applications for 'abled' users. As mentioned in the introduction, an abled person will also meet situations where only part of his or her available skills can be used and where an added communication, movement or control modality would be helpful. Moreover, having knowledge about the mental state of the user allows the application to adapt to it, leading to better performance. But, the applications can be designed in such a way that the user can always enter a built-in non-BCI mode where he or she uses the more traditional modalities for control and interaction.

Game designers have even more freedom. They can translate shortcomings in current BCI technology in challenges that need to be solved by a gamer and in decision moments that explicitly require a gamer to issue BCI commands, whether it is motor imagery, externally evoked potentials or by control of global brain activity. In game applications we can be satisfied with far from perfect 'solutions' since the game can be adapted to state-of-the-art theory and technology and nevertheless be interesting and challenging. Or maybe, because of such constraints, be even more challenging than when offering a gamer perfect BCI technology. We can have perfect game applications with imperfect technology.

Until now, in the academic BCI research community there are no or only modest research attempts to integrate BCI in multimodal interaction research and applications. Attempts to do so do not help ALS, Parkinson or epileptic patients. Now that BCI is becoming part of computer science and human-computer interaction research, multimodality is becoming an issue and there is both a 'technology push' to, and a 'technology pull' from potential application areas outside the medical domain. Nevertheless there is a controversy. Academics tell the industry that there are still lots of fundamental research issues that have to be investigated before a game with embedded intelligence and autonomous game agents can be developed and designed. Industry knows that imperfect interaction models and imperfect interaction technologies do not prevent the development of highly successful games. 
As an example, consider the design of an air-traffic control system, the design of a remote surgery system, or the design of a crisis management system. Such systems aim at preventing, avoiding and managing surprise situations. The aim is to have a system that, as far as is possible, protects the user from making wrong decisions. In a game the situation is different. Games are meant to provide the gamer with challenges. A game designer should take care that gamers can match their skills with these challenges and they should take care that skills that are improved during game playing will meet new and higher challenges. Game design is challenging the gamer in such a way that the gamer remains convinced that his or her skills can match these challenges or can be sufficiently improved by spending more time on trying to meet these challenges. The game, the game environment, the game characters or other users in a multiplayer situation are opponents that try to force you to make wrong decisions rather than support you to make right decisions. A gamer is also allowed to lose.

Another view on the use of BCI in games is the following. Imagine a game situation where you have to fight an enemy. For example, you are engaged in a sword fight. You may try to kill your opponent. If you are not successful in your first attempt, try a second time. In a BCI game situation it is quite natural that BCI control is not immediately successful. You have to try a second time, for example by imaging a certain movement, making your opponent weaker by issuing a blow, or a third time, making your opponent even weaker, before, finally, in a fourth attempt, you are successful in eliminating your opponent. This is a rather acceptable and natural sequence of events in a game environment, but this is also a rather undesirable and unacceptable sequence of events in an air-traffic control or a crisis management situation. The example shows that in games a game designer can create situations where BCI signals can be used in a natural game-life-like way, and not necessarily a real-life-like way. In games there is no need to be realistic or supportive, as long as the events and their control are believable in the game context and environment.

As mentioned before, in game environments BCI can complement other modalities rather than replace other modalities. Usually, in BCI experiments the researchers try to prevent and get rid of EMG (ElectroMyoGraphy) activity. This is electrical activity caused by muscle movement and participants of these experiments are asked or forced not to move. This kind of research is necessary and useful from the point of view of BCI research and applications where a patient is not able to move. When, however, we look at applications for healthy users it is also useful to investigate how EMG activity and information from other modalities can help to get a more complete picture of the user, his or her intentions in controlling a game situation, and his or her affective state. Moreover, unlike an application for a patient such as to recover control of muscles, to control a prosthesis or to communicate by thoughts alone with the outside world, for games we have the freedom to combine approaches such as motor imagery with real movements, or to use brain activity that is associated with real movements to control an application. In our experiments [15] we found support for the hypothesis that actual movement as modality in BCI games is better recognizable than imagined movements. It does not necessarily mean that people prefer actual movements, but this is of course a matter of game design.

In game situations we can also expect and stimulate measurable brain activity at particular moments. The game designer can design situations that require interaction 
between gamer and artificially evoked and event-related potentials. These evoked potentials can give a gamer control of situations and events, which can be purposely introduced when designing the game. Evoked potentials can be used to let the gamer make a decision based on events explicitly asking for attention. To use event-related potentials the gamer does not have to be in control. Naturally occurring events in the game or the actions of the gamer's opponent evoke surprise, anticipation and response reactions of the gamer and they can be employed in the game design too.

The main message of this section is that game designers have the freedom to combine different $\mathrm{BCI}$ and other modalities and that they have the freedom to do, from a BCI point of view, very unusual things. Until now this freedom has not been explored. In addition, apart from the multimodal approach, embedding BCI in contexts where artificial intelligence (reasoning, common sense knowledge, knowledge of user goals and preferences) is another road to follow for designing BCI games. We are certainly aware of the problems that are associated with distinguishing and fusing the signals that come from different sources. But, this does not change our view that what are considered to be shortcomings of BCI in traditional BCI applications can be seen as game challenges from a game design point of view.

\section{Developments and Challenges}

In previous sections we have already mentioned many of the problems that prevent the design of full-fledged BCI games now and in the near future. In this section we mention further problems that need technical solutions or that need to be circumvented in one or other way by clever game design.

\subsection{Multimodal Interaction and Artefacts}

BCI game research requires the integration of theoretical research on multimodal interaction, intention detection, affective state and visual attention monitoring, and on-line motion control, but also the design of several prototypes. These may be games for amusement, but also (serious) games for education, training and simulation.

There are many challenges unique to BCI applications in Human Computer Interaction. One challenge is the inevitable presence of artifacts deemed to be "noise" in traditional BCI explorations. In our applications, we cannot typically control the environment as tightly as in many medical applications (e.g. we do not want to be gaming in a Faraday Cage) nor are we willing to restrict the actions of the user (e.g. tie them down so they do not move). Hence, we have to devise techniques that either sidestep these issues, or better yet, leverage the additional information available. A particular point of interest is how to fuse information coming from more traditional input modalities (e.g. touch, speech, gesture) with information obtained from the brain. Consciously produced brain signals need to be distinguished from other brain activity. This other brain activity is produced because the gamer is involved in other activities at the same time (responses to visual, auditory and tactile channels), because of physiological responses, because of movements needed to play the game because of natural movements beyond or almost beyond control of the gamer. 


\subsection{Measuring Brain Signals}

In current BCI research EEG caps with 32 to 256 electrodes are used to measure brain activity. EEG is combined with EMG techniques as well, where the latter is about electrical activity that is obtained from face and body muscle movements. Rather than being happy with additional sources of information and including them in their research, BCI researchers often aim at pure mental BCI interfaces and consider the influence of muscle and eye movements as artifacts that have to be discounted.

We cannot expect that current EEG caps used in research (Figure 3a) will become part of a gamer's equipment. In addition to being expensive, for games we will not need up to 256 electrodes measuring activity from various parts of the brain. Moreover, setting up such a BCI session takes too much time, requiring the use of conductive gel, careful positioning of electrodes, and clean-up afterwards. But, both in research and commercial environments we see the development of 'dry-cap' technology. Experiments that investigate the consequences of using less electrodes and using a 'dry-cap' are reported in [27]. They report placing six electrodes at sites above the motor cortex and achieving 70 percent of full-gel-cap BCI performance.
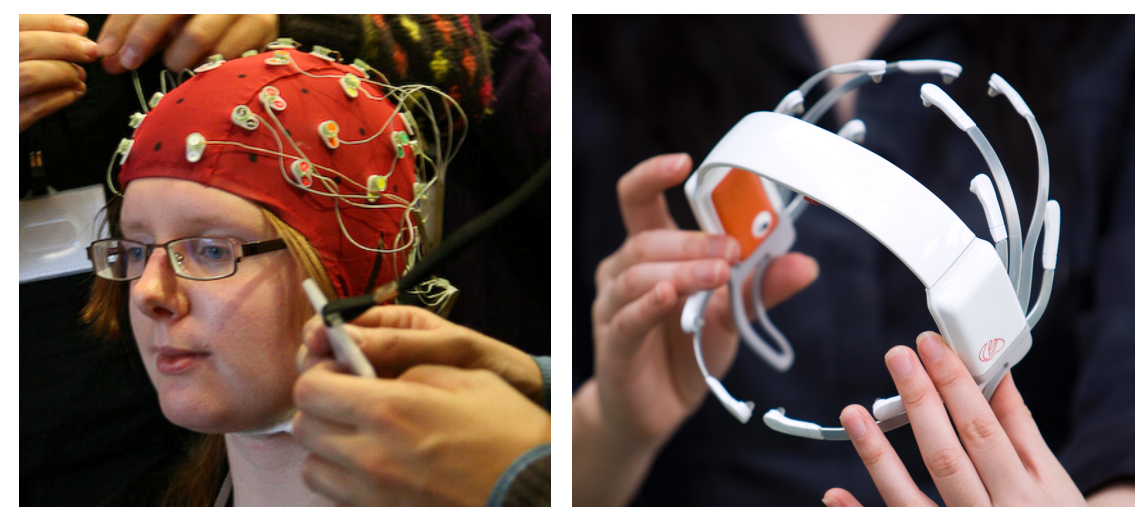

Fig. 3. a) BioSemi EEG cap for research, and b) Emotiv EEG headset for gaming [7]

Clearly, game companies are interested in this technology. They are also aware of the effect of a beautifully designed cap on gamers and the game community. Hence, can a cap be designed to have similar effects as owning and using an iPod, an iPhone, or a Wii? Or, for more business-like applications, using a wearable microphone or teleconferencing equipment? The effect that design has on user acceptance should not be underestimated. The interest in the BCI company Emotiv is certainly due to the futuristic headset they offer (Figure 3b). It resembles a headset used in the cult movie Strange Days that is used to access earlier recorded memories and emotions. In such a headset other features that are interesting for gamers can be included. As mentioned earlier, the Emotiv headset also measures head movements that can provide input to the game. Adding a microphone to record speech commands is another possibility.

An alternative for EEG measuring is fNIR (functional Near-Infrared imaging). Changes in brain activity are accompanied with blood flow. By firing near-infrared 
rays into the brain and converting the reflected light to electrical signals changes in oxygen concentrations in the blood can be measured allowing one to draw conclusions about the brain activity in various regions of the brain. NIRS techniques have been used to power switches on and off by doing mental arithmetic. At this moment wearing the equipment required to do NIR measuring is certainly far away from applications outside the research laboratory. But also here, with an iPod and Wii user market in mind, unexpected things may happen.

Measuring brain signals from outside the scalp using EEG is far from ideal. Placing sensors directly on the brain would provide stronger and more accurate signals but this would require surgery. Nevertheless, we can expect that in the future this and more invasive surgery will be performed to extend human capabilities for non-medical applications, including games and entertainment.

\subsection{Training and Illiteracy}

Issues that should be mentioned when we look at measuring brain activity in order to use it to give commands to the game environment are training and BCI illiteracy. Looking at training, in research environments, during a training period, both the user and the system learns. The particular EEG patterns that are produced by one particular user are training material for the BCI system. Users, on the other hand, learn to modulate their brain patterns, by getting feedback about their performance, allowing the application to distinguish them and to map them on the commands that control the application. Users and BCI adapt to each other [11,27]. Users can be asked to perform different mental tasks in order to generate various brain commands. Although the BCI environment can help in strongly suggesting such mental tasks, it cannot fully control the way a user performs a task. In commercial games we sometimes see that up to thirteen game control commands have to be distinguished.

Rather than go through a training and calibration period that allows a BCI game application to get acquinted with a particular user, we foresee the development of game applications where training becomes part of the game, requiring the game designer to introduce game elements and game levels that require BCI activity to handle challenging game situations. Adding BCI to games fits in the current developments to exploit games that use all kinds of sensors and algorithms that know about speech characteristics, about facial expressions, gestures, location and identity of the gamer and physiological processes that can be used to adapt or control the game. Similar observations have been made for so-called exertion interfaces [23] where users are assumed to be engaged in physical activity and where it is suggested to include a 'warming-up' in the sports/fitness/exercise environment [32].

From research experiments we can learn that not all BCI users are able to perform at the same level. That is, not all users in these experiments are able to imagine movements or perform other mental tasks in such a way that a BCI system is able to detect them in a sufficiently reliable way, that is, allowing the system to interprete a particular and measurable brain activity and converting it into a particular command that affects the environment and its inhabitants. Performance is highly subjectspecific. In [27] experiments are reported on motor imagery where 20 percent of the subjects show no effective motor imagery and where another 30 percent exhibit (too) slow (a) performance. 


\section{Conclusions}

In this paper we surveyed the 'state of the art' of brain-computer interfacing in the context of games and entertainment. It should be clear from this survey that 'games and BCI' is an exciting topic. There are various possibilities in game design to provide a gamer with ways to control game situations using BCI and to add to other input modalities in order to modify them to control game situations. Games can be designed in such a way that game control by thought is possible and that game environments know how to adapt to cognitive and motoric skills of the gamer. Game companies involved in these activities (Microsoft, Emotiv, Hitachi, Sega Toys, IBM, etc.) are keen on filing patents and are very reluctant to share their ways of thinking about the BCI modality in games. From this survey it should be clear that at this moment there are many unexplored roads of research on the role of BCI in game research and game design.

Acknowledgments. This work has been supported by funding from the Dutch National SmartMix project BrainGain [28] on BCI (Ministry of Economic Affairs) and the GATE project, funded by the Netherlands Organization for Scientific Research (NWO) and the Netherlands ICT Research and Innovation Authority (ICT Regie). We made use of our earlier work [29,33] to compile the survey on BCI games that is part of this paper.

\section{References}

1. Bartolo, A., Benuzzi, F., Nocetti, L., Baraldi, P., Nichelli, P.: Humor Comprehension and Appreciation: An fMRI Study. Journal of Cognitive Neuroscience 18(11), 1789-1798 (2006)

2. Bayliss, J.D.: Use of the evoked potential p3 component for control in a virtual apartment. IEEE Transactions on Neural Systems and Rehabilitation Engineering 11(2), 113-116 (2003)

3. Bayliss, J.D., Inverso, S.A., Tentler, A.: Changing the P300 Brain Computer Interface. CyberPsychology \& Behavior 7(6), 694-704 (2004)

4. Cairns, P., Cox, A., Berthouze, N., Dhoparee, S., Jennett, C.: Quantifying the experience of immersion in games. In: Cognitive Science of Games and Gameplay workshop at Cognitive Science (2006)

5. Csikszentmihalyi, M.: Flow: the psychology of optimal experience. Harper \& Row, New York (1990)

6. De Mey, T.: Tales of the Unexpected: Incongruity-Resolution in Humor Comprehension, Scientific Discovery and Thought Experimentation. Logic and Logical Philosophy 14, 6988 (2005)

7. Emotiv Systems, http://www.emotiv.com

8. FitzPatrick, I.: Effects of sentence context in L2 natural speech comprehension. Proc. of the Cognitive Neuroscience Master of the Radbout University 2(1), 43-56 (2007)

9. FUGA, Fun of Gaming: Measuring the Human Experience of Media Enjoyment. Project funded by the European Commission under the 6th Framework Programme: New and Emerging Science and Technology (NEST) (2006),

http: //project.hkkk. fi/fuga/ 
10. Gilleade, K., Dix, A., Allanson, J.: Affective Videogames and Modes of Affective Gaming: Assist Me, Challenge Me, Emote Me. In: Proc. DIGRA 2005 (2005)

11. Guger, C., Edlinger, G.: How many people can control a brain-computer interface (BCI)? In: Nijholt, A., Tan, D. (eds.) Proc. BrainPlay 2007: Playing with Your Brain. Workshop Intern. Conf. on Advances in Computer Entertainment Technology (ACE 2007), pp. 29-32 (2007)

12. Hjelm, S.I., Browall, C.: Brainball - Using brain activity for cool competition. In: Proceedings of NordiCHI (2000)

13. KEIO, http://www.bme.bio.keio.ac.jp/eng/01news/

14. Krepki, R., Blankertz, B., Curio, G., Müller, K.R.: The Berlin Brain-Computer Interface (BBCI)-towards a new communication channel for online control in gaming applications. Multimedia Tools and Applications 33(1), 73-90 (2007)

15. van de Laar, B., et al.: Actual and imagined movemnet in BCI gaming: Actually different? Manuscript, Human Media Interaction group, University of Twente, the Netherlands (December 2008)

16. Lalor, E., Kelly, S.P., Finucane, C., Burke, R., Reilly, R.B., McDarby, G.: Brain Computer Interface based on the Steady-State VEP for Immersive Gaming Control. In: Graz BCI Workshop (2004)

17. Leeb, R., Scherer, R., Lee, F., Bischof, H., Pfurtscheller, G.: Navigation in virtual environments through motor imagery. In: 9th Computer Vision Winter Workshop, CVWW, vol. 4, pp. 99-108 (2004)

18. Lehtonen, J., Jylanki, P., Kauhanen, L., Sams, M.: Online Classification of Single EEG Trials During Finger Movements. IEEE Trans. on Biomed. Eng. 55(2 Pt 1), 713-720 (2008)

19. Lin, T.A., John, L.R.: Quantifying Mental Relaxation with EEG for use in Computer Games. In: International Conference on Internet Computing, pp. 409-415 (2006)

20. Lotze, M., Halsband, U.: Motor imagery. Journal of Physiology 99(4-6), 386-395 (2006)

21. Martinez, P., Bakardjian, H., Cichocki, A.: Fully online multi-command brain-computer interface with visual neurofeedback using SSVEP paradigm. Computational Intelligence and Neuroscience 2007(1), 13 (2007)

22. Middendorf, M., McMillan, G., Calhoun, G., Jones, K.S.: Brain-computer interfaces based on the steady-state visual-evoked response. IEEE Transactions on Rehabilitation Engineering 8(2), 211-214 (2000)

23. Müller, F., Agamanolis, S., Picard, R.: Exertion Interfaces: Sports over a Distance for Social Bonding and Fun. In: ACM Conference on Human Factors in Computing Systems (CHI 2003), pp. 561-568 (2003)

24. Müller-Putz, G., Scherer, R., Pfurtscheller, G.: Game-like Training to Learn Single Switch Operated Neuroprosthetic Control. In: BRAINPLAY 2007 Brain-Computer Interfaces and Games Workshop at ACE (Advances in Computer Entertainment) (2007)

25. Nijholt, A.: Playing and Cheating in Ambient Entertainment. In: Ma, L., Rauterberg, M., Nakatsu, R. (eds.) ICEC 2007. LNCS, vol. 4740, pp. 415-420. Springer, Heidelberg (2007)

26. Nijholt, A., Tan, D., Allison, B., del, J., Millán, R., Graimann, B., Jackson, M.M.: BrainComputer Interfaces for HCI and Games. In: Proceedings ACM CHI 2008: Art. Science. Balance, Florence, Italy, pp. 3925-3928. ACM Publishing, NY (2008)

27. Nijholt, A., Tan, D., Pfurtscheller, G., Brunner, C., Del, J., Millan, R., Allison, B., Graimann, B., Popescu, F., Blankertz, B., Müller, K.-R.: Brain-Computer Interfacing for Intelligent Systems. IEEE Intelligent Systems, pp. 76-83 (May/June 2008) 
28. Nijholt, A., van Erp, J., Heylen, D.K.J.: BrainGain: BCI for HCI and Games. In: Proceedings AISB Symposium Brain Computer Interfaces and Human Computer Interaction: A Convergence of Ideas, Aberdeen, UK, April 2, pp. 32-35 (2008)

29. Oude Bos, D.: BrainBasher: A multi-modal BCI game for research and demonstration. Masters Thesis, HMI, University of Twente, the Netherlands (September 2008)

30. Pineda, J.A., Silverman, D.S., Vankov, A., Hestenes, J.: Learning to control brain rhythms: making a brain-computer interface possible. IEEE Transactions on neural systems and rehabilitation engineering 11(2), 181-184 (2003)

31. Pope, A.T., Palsson, O.S.: Helping Video Games, Rewire our Minds (manuscript, 2004)

32. Powell, V.: CHI 2008 Exertion Interfaces: A Flexible approach. In: Exertion Workshop at CHI 2008, Florence (2008)

33. Reuderink, B.: Games and Brain-Computer Interfaces: The State of the Art. WP2 BrainGain Deliverable, HMI, University of Twente (September 2008)

34. Scherer, R., Schloegl, A., Lee, F., Bischof, H., Jan`sa, J., Pfurtscheller, G.: The Self-Paced Graz Brain-Computer Interface: Methods and Applications. Computational Intelligence and Neuroscience, Article ID 79826 (2007)

35. Shim, B.-S., Lee, S.-W., Shin, J.-H.: Implementation of a 3-Dimensional Game for developing balanced Brainwave. In: 5th ACIS International Conference on Software Engineering Research, Management \& Applications (SERA 2007), pp. 751-758 (August 2007)

36. Strehl, U., Leins, U., Goth, G., Klinger, C., Hinterberger, T., Birbaumer, N.: Selfregulation of Slow Cortical Potentials: A New Treatment for Children With AttentionDeficit/Hyperactivity Disorder, Pediatrics (2006)

37. Vidal, J.J.: Real-time detection of brain events in EEG. Proceedings of the IEEE 65(5), 633-641 (1977) 